ERRATUM

M. Z. Liu • G. M. Jiang • Y. G. Li • S. L. Niu •

L. M. Gao $\cdot$ L. Ding $\cdot$ Y. Peng

\title{
Leaf osmotic potentials of 104 plant species in relation to habitats and plant functional types in Hunshandak Sandland, Inner Mongolia, China
}

Published online: 18 January 2005

(C) Springer-Verlag 2005

\section{Trees (2003) 17:554-560}

Errors have arisen in Table 2 on P. 556 and relative to Fig. 2 on P. 558 with respect to photosynthetic pathways. We present here the corrected Table 2 and Fig. 2.

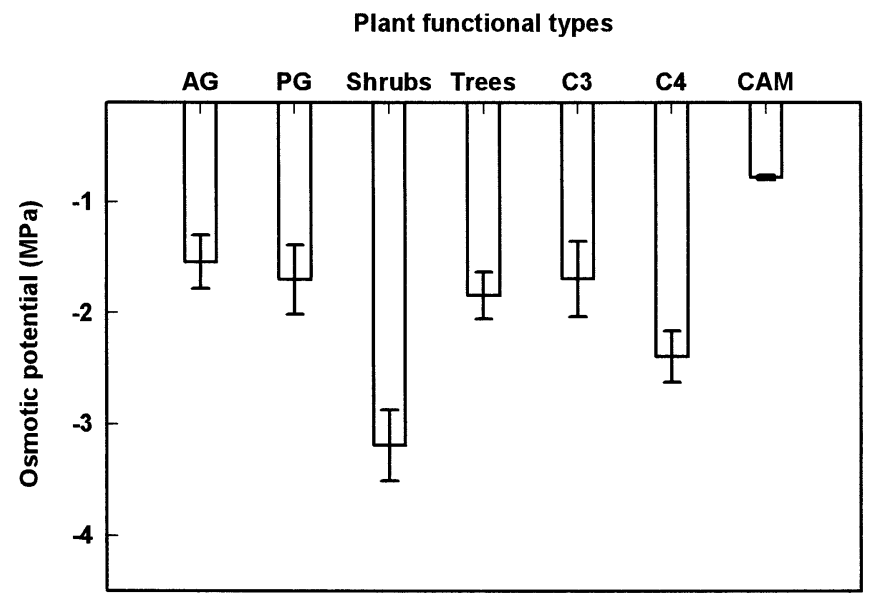

Fig. 2. Osmotic potentials of different plant functional types (PFTs) in Hunshandak ( $A G$ annual grasses, $P G$ perennial grasses). The mean values of all plants with the same PFT are presented

\section{References}

Watson L, Dallwitz MJ. (1992) The grass genera of the world. CAB International, Wallingford, Oxon OX 108 DE, UK

The online version of the original article can be found at http://dx. doi.org/10.1007/s00468-003-0277-8

M. Z. Liu · G. M. Jiang $(\bowtie) \cdot$ Y. G. Li · S. L. Niu · L. M. Gao ·

L. Ding · Y. Peng

Laboratory of Quantitative Vegetation Ecology, Institute of

Botany, The Chinese Academy of Sciences,

20 Nanxincun, Xiangshan,

100093 Beijing, P.R. China

e-mail: jgm@ht.rol.cn.net

Tel.: +86-10-010625914316286

Fax: +86-10-01062590843 
Table 2. Leaf osmotic potentials $\left(\psi_{\mathrm{s}}\right)$ and roots depth of different different habitats ( $F$ fixed sand dunes, $L$ lowland, $W$ wetland) in the plant species with different growth forms ( $G$ grasses, $S$ shrubs, $T$ Hunshandak Sandland in summer 2001. The means of three trees), different photosynthetic pathway $\left(C_{3}, C_{4}\right.$ and $\left.C A M\right)$ on measurements are reported $\pm \mathrm{SD}$

\begin{tabular}{|c|c|c|c|c|c|c|c|}
\hline Family & Species & $\begin{array}{l}\text { Growth } \\
\text { form }\end{array}$ & $\begin{array}{c}\text { Photosynthetic } \\
\text { pathway }\end{array}$ & Habitats & $\begin{array}{l}\text { Root depth } \\
\quad(\mathrm{cm})\end{array}$ & $\psi_{\mathrm{s}}(\mathrm{MPa})$ & $\begin{array}{c}\delta{ }^{13} \mathrm{C}(\%) \\
\mathrm{PDB}\end{array}$ \\
\hline Gramineae & Digitaria ischaemum Schreb. & G & $\mathrm{C}_{4}{ }^{*}$ & W & 8 & $-0.44 \pm 0.21$ & \\
\hline Scrophulariaceae & Linaria vulgaris Mill. & $\mathrm{G}$ & $\mathrm{C}_{3}$ & $\mathrm{~L}$ & 10 & $-1.20 \pm 0.07$ & \\
\hline Gramineae & Leymus chinensis (Trin.) Tzvel. & G & $\mathrm{C}_{3} *$ & $\mathrm{~F}$ & 10 & $-2.24 \pm 0.14$ & \\
\hline Compositae & Inula britannica $\mathrm{L}$. & G & $\mathrm{C}_{3}$ & $\mathrm{~F}$ & 12 & $-1.32 \pm 0.13$ & \\
\hline Campanulaceae & Campanula rotundifolia $\mathrm{L}$. & G & $\mathrm{C}_{3}$ & $\mathrm{~L}$ & 14 & $-1.24 \pm 0.11$ & \\
\hline Chenopodiaceae & Chenopodium glaucum $\mathrm{L}$. & G & $\mathrm{C}_{3}$ & F & 20 & $-2.06 \pm 0.32$ & -29.75 \\
\hline Rosaceae & Potentilla strigosa Pall. & G & $\mathrm{C}_{3}$ & $\mathrm{~F}$ & 20 & $-2.30 \pm 0.14$ & \\
\hline Ranunculaceae & Halerpestes ruthenica (Jacq.) Ovcz. & G & $\mathrm{C}_{3}$ & $\mathrm{~L}$ & 24 & $-1.54 \pm 0.03$ & \\
\hline Gramineae & Agropyron cristatum (L.) Gaertn. & G & $\mathrm{C}_{3} *$ & F & 25 & $-2.02 \pm 0.14$ & -25.74 \\
\hline Rosaceae & Potentilla anserina $\mathrm{L}$. & G & $\mathrm{C}_{3}$ & $\mathrm{~F}$ & 25 & $-2.27 \pm 0.35$ & \\
\hline Compositae & Artemisia aпnиa $\mathrm{L}$. & G & $\mathrm{C}_{3}$ & $\mathrm{~F}$ & 28 & $-1.82 \pm 0.19$ & \\
\hline Umbelliferae & Ferula bungeana Kitag. & G & $\mathrm{C}_{3}$ & $\mathrm{~F}$ & 30 & $-0.84 \pm 0.09$ & \\
\hline Labiatae & $\begin{array}{l}\text { Thymus serpyllum L.var.mongolicum } \\
\text { Roem }\end{array}$ & G & $\mathrm{C}_{3}$ & $\mathrm{~F}$ & 30 & $-0.87 \pm 0.27$ & \\
\hline Compositae & Heteropappus hispidus Willd. & G & $\mathrm{C}_{3}$ & $\mathrm{~F}$ & 30 & $-1.81 \pm 0.78$ & \\
\hline Chenopodiaceae & Bassia dasyphylla (Fisch.) O. Kuntze & G & $\mathrm{C}_{3}$ & $\mathrm{~F}$ & 30 & $-2.48 \pm 0.12$ & -25.19 \\
\hline Umbelliferae & Cnidium monnieri (L.) Cuss. & G & $\mathrm{C}_{3}$ & $\mathrm{~F}$ & 38 & $-1.89 \pm 0.05$ & \\
\hline Amaranthaceae & Amaranthus retroflexus $\mathrm{L}$. & G & $\mathrm{C}_{4}$ & $\mathrm{~F}$ & 40 & $-1.47 \pm 0.18$ & -15.60 \\
\hline Gramineae & Leymus secalinus (Georgi) Tzvel. & G & $\mathrm{C}_{3}$ * & $\mathrm{F}$ & 40 & $-3.05 \pm 0.18$ & \\
\hline Urticaceae & Urtica cannabina $\mathrm{L}$. & G & $\mathrm{C}_{3}$ & $\mathrm{~F}$ & 46 & $-1.92 \pm 0.47$ & \\
\hline Caryophyllaceae & Silene repens var.angustifolia Turcz. & G & $\mathrm{C}_{3}$ & $\mathrm{~F}$ & 50 & $-2.29 \pm 0.36$ & -26.72 \\
\hline Umbelliferae & Bupleurum sibiricum Vest. & G & $\mathrm{C}_{3}$ & $\mathrm{~F}$ & 55 & $-2.30 \pm 0.73$ & \\
\hline Gramineae & Cleistogenes squarrosa (Trin.) Keng & G & $\mathrm{C}_{4} *$ & $\mathrm{~F}$ & 60 & $-1.96 \pm 0.22$ & -15.39 \\
\hline Leguminosae & Medicago falcata $\mathrm{L}$. & G & $\mathrm{C}_{3}$ & $\mathrm{~F}$ & 70 & $-1.97 \pm 0.28$ & \\
\hline Chenopodiaceae & Agriophyllum pungens (Vahl) Link & G & $\mathrm{C}_{3}$ & F & 90 & $-1.40 \pm 0.08$ & -26.96 \\
\hline Leguminosae & Amorpha fruticosa $\mathrm{L}$. & $\mathrm{T}$ & $\mathrm{C}_{3}$ & $\mathrm{~F}$ & 100 & $-1.96 \pm 0.20$ & \\
\hline Salicaceae & Salix microstachya & $\mathrm{S}$ & $\mathrm{C}_{3}$ & F & 120 & $-2.6 \pm 0.15$ & \\
\hline
\end{tabular}

* Watson and Dallwitz (1992)

${ }^{*} \delta^{13} \mathrm{C}$ values are unpublished data by courtesy of Hubert Ziegler, Munich, Germany 